\title{
Properties of X-ray emission of an aspherical shock breakout
}

\author{
Yukari Ohtani $^{1}$, Akihiro Suzuki ${ }^{2}$, Toshikazu Shigeyama ${ }^{3}$ \\ and Masaomi Tanaka ${ }^{4}$ \\ ${ }^{1}$ Center for Computational Astrophysics, National Astronomical Observatory of Japan, \\ Mitaka, Tokyo 181-8588, Japan \\ email: yukari.ohtani@nao.ac.jp \\ ${ }^{2}$ Yukawa Institute for Theoretical Physics, Kyoto University, \\ Oiwake-cho, Kitashirakawa, Sakyo-ku, Kyoto, 606-8502, Japan \\ email: asuzuki@kusastro.kyoto-u.ac.jp \\ ${ }^{3}$ Research Center for the Early Universe, Graduate School of Science, University of Tokyo, \\ Bunkyo-ku, Tokyo 113-0033, Japan \\ email: shigeyama@resceu.s.u-tokyo.ac.jp \\ ${ }^{4}$ Division of Theoretical Astronomy, National Observatory of Japan, \\ Osawa 2-21-1, Mitaka, Tokyo 181-8588, Japan \\ email: masaomi.tanaka@nao.ac.jp
}

\begin{abstract}
We investigate the relation between the emission properties of supernova shock breakout in the circumstellar matter (CSM) and the behavior of the shock. Using a MonteCarlo method, we examine how the light curve and spectrum depends on the asphericity of the shock and bulk-Compton scattering, and compare the results with the observed properties of X-ray outburst (XRO) 080109/SN 2008D. We found that the rise and decay time of the X-ray light curve do not significantly depend on the degree of shock asphericity and the viewing angle in a steady and spherically symmetric CSM. The observed X-light curve and spectrum of XRO 080109 can be reproduced by considering the shock with a radial velocity of $60 \%$ of the speed of light, and the wind mass loss rate is about $5 \times 10^{-4} M_{\odot}$.
\end{abstract}

Keywords. radiative transfer, scattering, methods: numerical, supernovae: general, supernovae: individual (SN 2008D), X-rays: individual (XRO 080109)

\section{Introduction}

A core-collapse supernova becomes bright in ultraviolet/optical bands when photons generated from the shock diffuse upstream. The timescale of the phenomenon, so-called "shock breakout", is determined by the light crossing time of the size of the emerging shock and the diffusion time of photons (e.g., Ensman \& Burrows 1992; Matzner \& McKee 1999). Since the emission properties of shock breakout strongly depend on the behavior of the shock, they can be used as probes of the stellar radius and the structure of the stellar surface.

X-ray outburst (XRO) 080109 is a fortunately detected shock breakout (e.g., Soderberg et al. 2008) associated with a Type Ib SN 2008D. The luminosity (in the energy range of $[0.3,10] \mathrm{keV}$ ) reached its peak $6 \times 10^{43} \mathrm{erg} \mathrm{s}^{-1}$ at $\sim 100 \mathrm{sec}$ (hereafter $\Delta t_{\mathrm{p}}$ ) from the onset and decreased until $\sim 600$ sec. The spectrum taken by the Swift/XRT can be well fitted by a power-law distribution.

The timescale of XRO 080109 depends on the shock radius at the moment of breakout. The rise time can be interpreted as the light crossing time of the shock radius at the 
moment of breakout (Soderberg et al. 2008) or the shock expansion timescale (Svirski \& Nakar 2014). In both of the scenarios, the shock must break out at a radius of $\approx 10^{12}$ $\mathrm{cm}$, which is a larger than the typical radius of a Wolf-Rayed star. For this reason, the shock breakout is believed to occur in a dense circumstellar matter (CSM). The scenario is also consistent with the fact that the duration of XRO 080109 corresponds to the diffusion timescale of photons in the unshocked CSM (Balberg \& Loeb 2011). Since a wind mass loss event can cause uncertainties in the evolution of a massive star, studying shock breakout is also essential for stellar evolution shortly before the explosion.

The strong candidate of the origin of the observed power-law spectrum is repeated electron ("bulk-Compton") scattering across the shock. In fact, the observed spectrum of XRO 080109 can be reproduced by considering a shock with a radial velocity of $\sim 0.3 c$, where $c$ denotes the speed of light (Suzuki \& Shigeyama 2010a).

In addition to the signatures of shock breakout in the CSM and bulk-Comptonization, the possibility of the asphericity of the shock has also been discussed. If the shock breaks out in the vicinity of the stellar surface (Suzuki \& Shigeyama 2010a; Couch et al. 2011; Suzuki et al. 2016), the peak of the light curve is believed to be broader than that of a spherical symmetric supernova.

However, there have been no studies which reproduced both of the observed light curve and spectrum of XRO 080109 by an aspherical shock propagating into a thick CSM and bulk-Comptonization. In this study, we perform a radiative transfer calculation aiming to fully reconstruct the observation. In Section 2, we describe the model for the shock and how we calculate radiative transfer. In Section 3, we show the results and compare them with XRO 080109. In Section 4, we conclude this article.

\section{Methods}

We calculate the light curve and spectrum of the emission generated from the shock propagating into the CSM. In the following subsections, we describe our model for the shock (§2.1) and settings for radiative transfer calculations (§2.2).

\subsection{Model for shock}

To investigate the properties of X-ray emission, we use a simple model of an ellipsoidal shock in a steady and spherically symmetric wind as described in Figure 1 (left panel). The ejecta is regarded as a piston. The shocked ejecta is ignored because most photons are absorbed of scattered immediately at the contact surface where the supposed density is as high as $10^{20} \mathrm{~cm}^{-3}$.

The matter is radiation dominated (thus the adiabatic index equals $4 / 3$ ) behind the shock front. We calculate a pure radiation transfer in the shocked matter, so that the feedback from radiation is not taken into account. Here we consider a shock of which radial velocity does not change with time and follows the formula

$$
v(f, \theta)=\frac{1-f}{\left[(1-f)^{2} \cos ^{2} \theta+\sin ^{2} \theta\right]^{1 / 2}} \times v(f, 0),
$$

where $f$ is the oblateness of the shock front, $\theta$ the angle measured from the symmetric axis. If the kinetic energy of the ejecta is fixed, $v(f, 0)=\sqrt{3} \times v_{f=0} \times\left(2 f^{2}-4 f+3\right)^{-1 / 2}$. In this study, $v_{f=0}=0.6 c$. The shock radial velocity is displayed in Figure 1 (right panel) as a function of $\theta$.

The shock radius $R_{\mathrm{b}}$ at the moment of breakout can be estimated by the observed rise time $\Delta t_{\mathrm{p}}$ of $\mathrm{XRO} 080109$. For $f=0$, we assumed that $R_{\mathrm{b}}=c \Delta t_{\mathrm{p}}=3 \times 10^{12}$ $\mathrm{cm}$. We also assumed that the distance between the shock front and contact surface is 

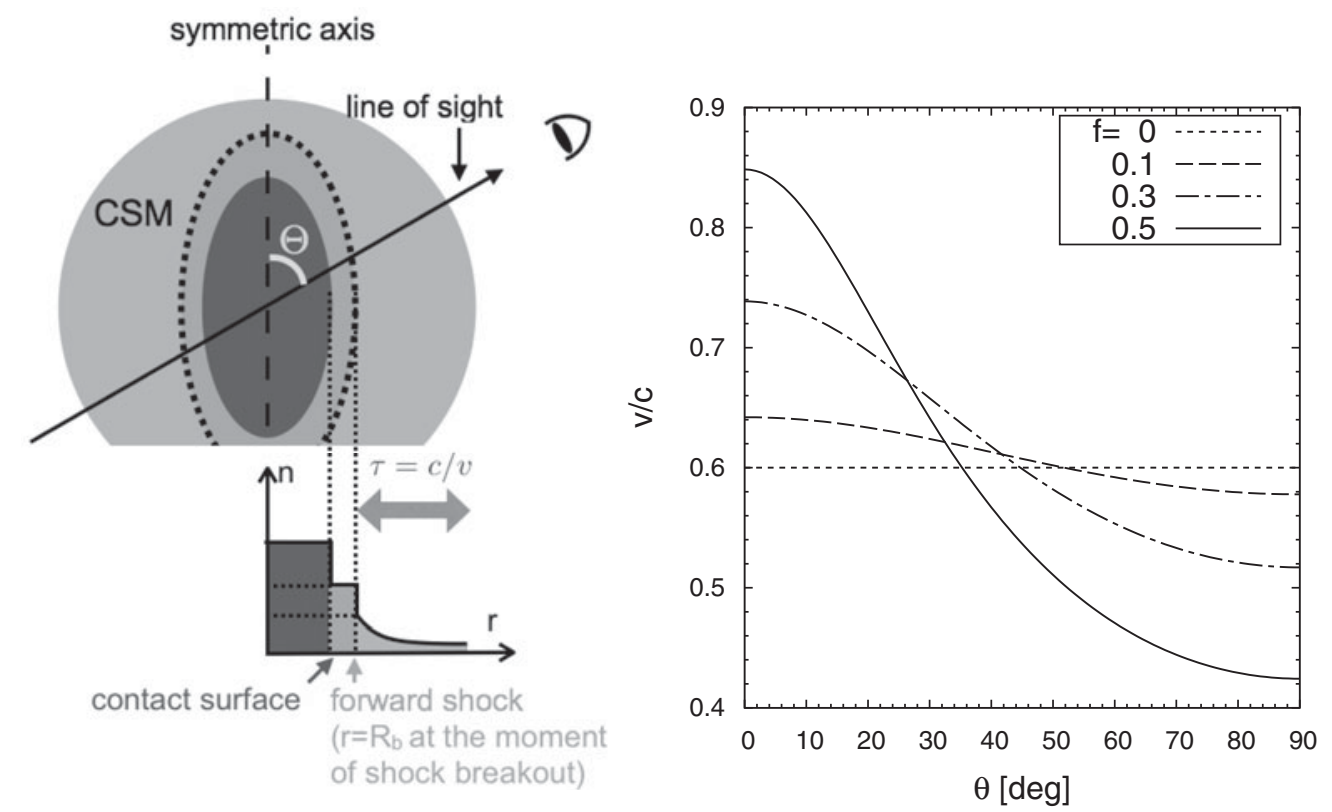

Figure 1. Left: Schematic view of an ellipsoidal shock driven by a piston moving in a steady, spherically symmetric CSM. Right: Angular distribution of the shock radial velocity for several values of oblateness $f$.

$R / 7$, where $R$ is the shock radius. In the unshocked CSM, the number density $n_{1}$ of electrons follows $n_{1}=A \times r^{-2}$ where $r$ is the radius. The constant $A$ is determined so that the optical depth equals $c / v(f, \theta)$ at $r=R_{\mathrm{b}}$. Under the assumption of $v_{f=0}=0.6 c$, $A=7.5 \times 10^{36} \mathrm{~cm}^{-1}$. The wind is supposed to emanate from a carbon-oxygen layer, and become stationary when its velocity decreases to $1,000 \mathrm{~km}$ thus the mass loss rate becomes $5 \times 10^{-4} M_{\odot}$. From the above constructions, the number density $n_{2}$ of electrons and temperature $k_{\mathrm{B}} T\left(k_{\mathrm{B}}\right.$ : the Boltzmann constant) become $\sim 10^{13} \mathrm{~cm}^{-3}$ and $0.11 \mathrm{keV}$ (hereafter $k_{\mathrm{B}} T_{\mathrm{b}}$ ) at the moment of shock breakout.

\subsection{Monte-Carlo method}

We calculate the transfer of photons generated from the shock front by using a MonteCarlo method. The basic construction of the code is the same as used in Ohtani et al. (2013). In this study, photons are assumed to be generated over a period of 0.5 sec with an energy distribution following the Planck function in the co-moving frame, and interact with matters via inverse Compton scattering and free-free absorption.

\section{Results}

First of all, we calculate the X-ray light curve and spectrum for a spherical shock and compare with the observation of XRO 080109. Then we investigate the dependence on the degree of shock asphericity and the viewing angle.

\subsection{Spherical shock}

The left panel of Figure 2 shows the X-ray light curve in the energy range of $[0.3,10]$ $\mathrm{keV}$ for the wind mass loss rate of $5 \times 10^{-4} M_{\odot} \mathrm{yr}^{-1}$ and the shock velocity of $0.6 \mathrm{c}$. The luminosity reaches its peak at $\sim 40 \mathrm{sec}$ and decreases for the for the subsequent several 

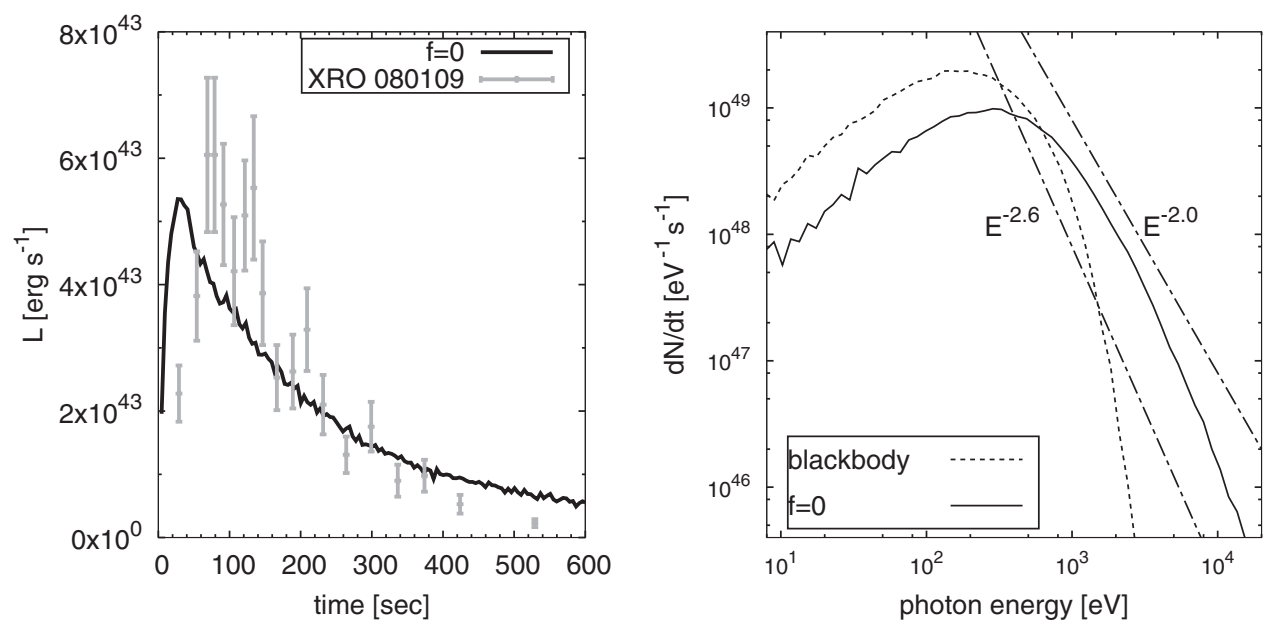

Figure 2. Left: Light curves in the energy range of $[0.3,10] \mathrm{keV}$ (left) compared with that of XRO 080109 (superposed bar) for $f=0$. Right: Time-integrated spectrum for $f=0$ (solid line) and a blackbody spectrum at a temperature of $k_{\mathrm{B}} T_{\mathrm{b}}$. The gradients $(-2.0$ and -2.6$)$ of the gray straight lines show the $\pm 1 \sigma$ values of those of XRO 080109 .

hundred seconds. The rise time mainly depends on the light crossing time $\Delta t_{\mathrm{lc}}$ of the size of the emerging shock and weakly on the diffusion timescale $\Delta t_{\text {diff }}^{\prime}(=\Delta R / v=110 \mathrm{sec})$ of photons in the shocked CSM. Due to weak relativistic effects, $\Delta t_{\mathrm{lc}}$ equals to $R_{\mathrm{b}}(1-$ $\left.\cos \theta_{\text {rel }}\right) / c=30 \mathrm{deg}$, where $\theta_{\text {rel }}=\tan ^{-1}\left(c \gamma^{-1} v^{-1}\right)=50 \mathrm{deg}$ and $\gamma=1 / \sqrt{1-(v / c)^{2}}$. The decay time is determined by the radius $R_{\text {final }}$ where photons last scatter off electrons. We can roughly estimate $R_{\text {final }}$ to be $\sim 10 R_{\mathrm{b}}$ where the optical depth of the outer CSM decreases down to $\sim 0.1$. Therefore the decay time $R_{\text {final }} / c$ equals to several hundred seconds. Note that we could not discuss the maximum luminosity because it depends on the assumed period of photon generation.

The right panel of Figure 2 shows the time-integrated spectrum (solid line) and a blackbody with a temperature of $k_{\mathrm{B}} T_{f=0}$ (dotted line). Due to the bulk-Comptonization, the maximum photon energy reaches $\sim m_{\mathrm{e}} v^{2} \sim 10 \mathrm{keV}$, where $m_{\mathrm{e}}$ is the electron mass. The high-energy component $(1-7 \mathrm{keV})$ can be fitted by a power-law distribution, of which gradient lies in the $1 \sigma$ error range of that of XRO 080109 (shown by straight lines in Figure 2).

\subsection{Aspherical shock}

We investigate the influence of the asphericity of the shock on the X-ray light curve and spectrum. In the previous works (Suzuki \& Shigeyama 2010a; Couch et al. 2011; Matzner et al. 2013; Salbi et al. 2014; Suzuki et al. 2016) the properties of the emission generated near the stellar surface were strongly by the asphericity of the shock. Our results are fairly different from that because the shock breaks out simultaneously in all directions under the assumption of a shock of a constant velocity and a steady and spherical wind.

Figure 3 shows the X-ray light curves $(0.3-10 \mathrm{keV})$ for aspherical shock waves. In the left panel, $f$ has several values $(0.1,0.3,0.5)$ and $\Theta=[80,90]$ deg (the flux is averaged over this angular range), and in the right panel, $f$ is fixed at 0.5 and $\Theta=[0,10]$, $[40,50]$ and $[80,90] \mathrm{deg}$. (The graphs are shifted to the left side so that the time of onset correspond to those for $f=0$.) The luminosity evolves with similar timescales (rise \& decay times) regardless of $f$. As with the spherically symmetric case, the decay time is uniquely determined by the density distribution of the CSM. The rise time corresponds 

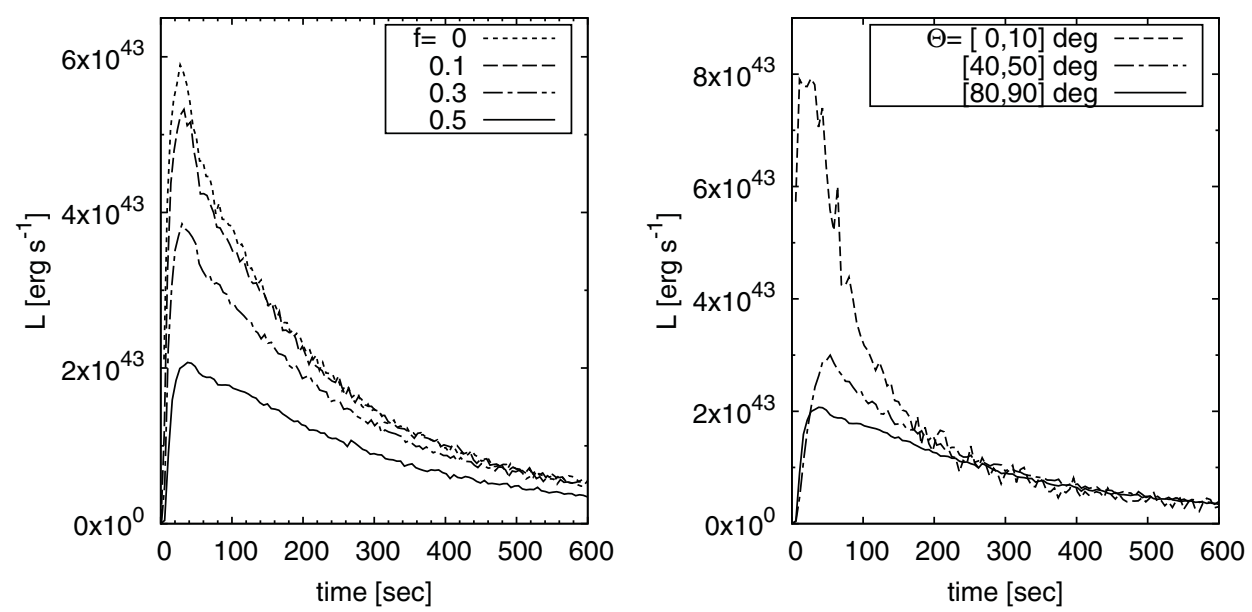

Figure 3. The same as the left panel of Figure 2 but for $f>0$. Left: $f$-dependence $(\Theta=[80,90]$ deg). Right: $\Theta$-dependence $(f=0.5)$. The graphs are shifted so that the times of onset correspond to those for $f=0$.


Figure 4. The same as Figure 2 but for $f=0.5$ and $\Theta=[40,50]$ deg.

to the light crossing time of the size of the emerging shock. If $f=0.5$ and $\Theta \sim 0 \operatorname{deg}$, for example, most photons traveling along the line of sight are generated near $(\theta \lesssim 30$ deg) the symmetry axis because of the high shock velocity $(\sim 0.8 \mathrm{c})$ along the line of sight. Therefore the rise time equals to $\sim\left[R_{\mathrm{b}, \theta=0}-R_{\mathrm{b}, \theta=30^{\circ}} \cos 30^{\circ}\right] / c \sim 40 \mathrm{deg}$, where $R_{\mathrm{b}, \theta=0}=4 \times 10^{12} \mathrm{~cm}$ and $R_{\mathrm{b}, \theta=30^{\circ}}=4 \times 10^{12} \mathrm{~cm}$. If $\Theta=90 \mathrm{deg}$, the influence of relativistic effects on the motion of photons becomes weak due to the lower shock velocity of $0.5 \mathrm{c}$. As a result, the rise time equals $\sim 40 \mathrm{sec}$, which is not significantly different from that for $\Theta=0$. The peak luminosity decreases with increasing $\Theta$, as well as the velocity of the shock propagating along the line of sight.

We compare the calculation for the ellipsoidal shock with the XRO 080109. Figure 4 shows the X-ray light curve (left) for $f=0.5$ and $\Theta=[40,50] \mathrm{deg}$, of which overall shape is roughly consistent with the observation. The spectral gradient, shown in the right panel of Figure 4, is also consistent with that of XRO 080109. An off-axis line of 
sight has been in fact suggested for XRO 080109 from the properties of nebular emission lines of SN 2008D (Tanaka et al. 2009).

\section{Conclusions}

In order to investigate the properties of X-ray emission from shock breakout in a dense CSM, we calculate transfer of X-ray photons interacting with matter through inverse Compton scattering and free-free absorption using a Monte-Carlo method. We also study relations between the asphericity of the shape of an ellipsoidal shock and the emission.

The rise time and duration of the X-ray light curve is mainly determined by the light crossing times of the size of the emerging shock and the radius where photons last scatter off electrons, respectively. The properties of the light curve (such as the rise time and duration) do not dramatically depend on the viewing angle and the degree of shock asphericity as long as a steady and spherically symmetric wind is considered. Both of the observed X-ray light curve and power-law spectrum of XRO 080109 can be reproduced by a shock with a radial velocity of $0.6 c$ and a (spherical) wind with a mass loss rate of $5 \times 10^{-4} M_{\odot}$.

\section{References}

Balberg, S. \& Loeb, A. 2011, MNRAS, 414, 1715

Couch, S. M., Pooley, D., Wheeler, J. C., \& Milosavljević, M. 2011, ApJ, 727, 104

Ensman, L. \& Burrows, A. 1992, ApJ, 393, 742

Matzner, C. D. \& McKee, C. F. 1999, ApJ, 510, 379

Matzner, C. D., Levin, Y., \& Ro, S. 2013, ApJ, 779, 60

Ohtani, Y., Suzuki, A., \& Shigeyama, T. 2013, ApJ, 777, 113

Salbi, P., Matzner, C. D., Ro, S., \& Levin, Y. 2014, ApJ, 790, 71

Soderberg, A. M., Berger, E., Page, K. L., Schady, P., Parrent, J., Pooley, D., Wang, X.-Y., Ofek, E. O., Cucchiara, A., Rau, A., Waxman, E., Simon, J. D., Bock, D. C.-J., Milne, P. A., Page, M. J., Barentine, J. C., Barthelmy, S. D., Beardmore, A. P., Bietenholz, M. F., Brown, P., Burrows, A., Burrows, D. N., Byrngelson, G., Cenko, S. B., Chandra, P., Cummings, J. R., Fox, D. B., Gal-Yam, A., Gehrels, N., Immler, S., Kasliwal, M., Kong, A. K. H., Krimm, H. A., Kulkarni, S. R., Maccarone, T. J., Mészáros, P., Nakar, E., O’Brien, P. T., Overzier, R. A., de Pasquale, M., Racusin, J., Rea, N., \& York, D. G. 2008, Nature, 453, 469

Suzuki, A. \& Shigeyama, T. 2010, ApJ, 717,154

Suzuki, A. \& Shigeyama, T. 2010, ApJ, 719,881

Suzuki, A., Maeda, K., \& Shigeyama, T. 2016, ApJ, 825, 92

Svirski, G. \& Nakar, E. 2014, ApJ, 788, 14

Tanaka, M., Yamanaka, M., Maeda, K., Kawabata, K. S., Hattori, T., Minezaki, T., Valenti, S., Della Valle, M., Sahu, D. K., Anupama, G. C., Tominaga, N., Nomoto, K., Mazzali, P. A., \& Pian, E. 2009, ApJ, 700, 1680 